\title{
Graphene is not alone
}

\author{
With the rise of two-dimensional transition metal dichalcogenides, graphene is no longer the only \\ two-dimensional crystal attracting significant interest in the research community.
}

The isolation of graphene from graphite by mechanical exfoliation in $2004^{1}$ showed that two-dimensional crystals could exist in a free-standing form. This single layer of carbon atoms - and its chemical modifications - has since become one of the most intensely studied materials, attracting the interest of physicists, chemists and engineers alike. As early as 2005, it was already clear that other layered materials could be exfoliated down to a monolayer ${ }^{2}$. But graphene has until recently remained the only two-dimensional crystal to receive widespread attention.

In the past two years, however, two-dimensional transition metal dichalcogenides (TMDCs), have shown intriguing physical properties and exciting prospects for a variety of applications. They are well-known materials, which are naturally found in layered forms like molybdenite or tungstenite. They have a very similar appearance to graphite and have applications primarily in solid lubricants. In the single-layer form some of these materials, such as molybdenum disulphide (a ball-and-stick model is shown; molybdenum, blue; sulphur, yellow), are direct bandgap semiconductors ${ }^{3,4}$, which could have important implications for their use in electronic devices.

In a Review in this issue ${ }^{5}$,

Qing Hua Wang et al. provide an overview of the properties of two-dimensional TMDCs, as well as their prospects for a wide range of applications, from electronics to chemical sensing. The presence of a gap in the electronic band is an essential requirement for the realization of common electronic devices like transistors. Finding the best way to induce such a gap in graphene has been a very active research topic in the past few years. In $\mathrm{MoS}_{2}$, the gap is intrinsic, and transistors and logic circuits based on single layers of this material have already been successfully demonstrated ${ }^{6,7}$. Such thin electronic devices cannot be achieved with commonly used materials like silicon, which tend to degrade when reduced in thickness.

The direct bandgap means that materials like $\mathrm{MoS}_{2}$ or $\mathrm{WS}_{2}$ could be used in lightemitting diodes, photodetectors or solar cells. Because of the thinness of these materials, the devices could be made transparent, light and flexible, and could provide a viable alternative to devices based on organic semiconductors, which degrade relatively quickly in ambient conditions. With a generous dose of optimism, we could even dream of flexible lasers based on TMDCs.

While efforts to develop devices based on twodimensional TMDCs are ongoing, another exciting strategy that is rapidly emerging is to combine different two-dimensional crystals in artificially produced layered materials. The idea here is simply to exfoliate layered materials and fabricate mixed stacks. It was recently shown that this procedure generates structures with clean atomic interfaces ${ }^{8}$, and tunnelling transistors have been fabricated using this strategy 9 . In principle, a whole new range of devices could be created by properly combining highly conductive graphene, insulating boron nitride and semiconducting $\mathrm{MoS}_{2}$ or $\mathrm{WS}_{2}$ (ref. 10).

There are, however, significant obstacles to overcome before any of these devices can become practical. For example, it is still unclear how two-dimensional TMDCs can be doped, either with electrons or holes. For proof-of-principle devices, electrical injection through gates is a possible route, but for commercial devices chemical doping will be necessary. Then there is the necessity of obtaining large quantities of high-quality single or few-layer materials in a cost-effective way. As noted by Wang et al. ${ }^{5}$, promising results have been achieved through chemical vapour deposition and liquid exfoliation, but more needs to be done.

It's not all about applications of course. The materials have already shown interesting properties, like valley polarization ${ }^{11,12}$. Moreover, a purely two-dimensional semiconductor may allow interesting fundamental studies of the properties of excitons (electron-hole pairs). For example, if a layer of $\mathrm{MoS}_{2}$ was inserted in a properly designed photonic cavity it would be possible to study the exciton polaritons coupled exciton-photon modes - in

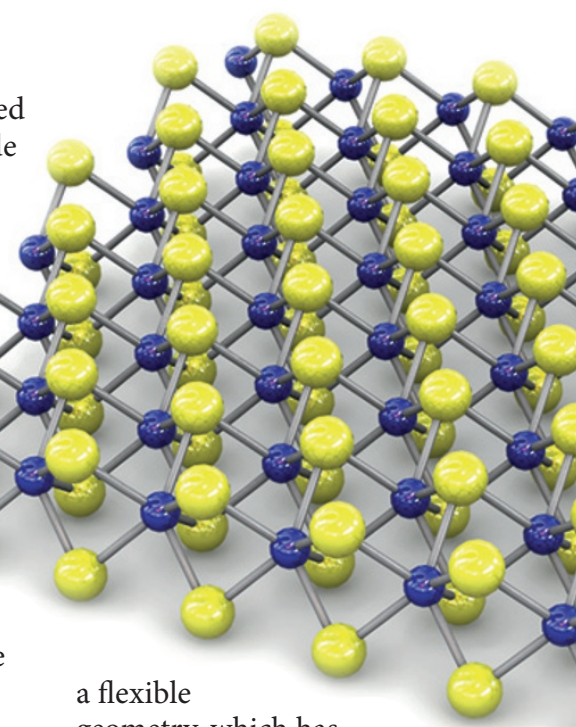

geometry, which has

never been achieved before.

It has taken a few years, but

two-dimensional TMDCs are now at the centre of attention of a wide community of scientists and engineers along with graphene and its chemical modifications. The original paper ${ }^{2}$ on the exfoliation of layered materials also reported layers of the hightemperature superconductor $\mathrm{Bi}_{2} \mathrm{Sr}_{2} \mathrm{CaCu}_{2} \mathrm{O}_{x}$ that were only one-unit-cell thick. Although no superconductivity was observed, further work on the processing of the material could perhaps allow the properties of a free-standing superconducting unit cell to be studied. So, stay tuned, for more two-dimensional materials to come of age.

\section{References}

1. Novoselov, K. S. et al. Science 306, 666-669 (2004).

2. Novoselov, K. S. et al. Proc. Natl Acad. Sci. USA 102, 10451-10453 (2005).

3. Li, T. \& Galli, G. J. Phys. Chem. C 111, 16192-16196 (2007).

4. Lebegue, S. \& Eriksson, O. Phys. Rev. B 79, 115409 (2009).

5. Wang, Q. H., Kalantar-Zadeh, K., Kis, A., Coleman. J. N. \& Strano, M. S. Nature Nanotech. 7, 699-712 (2012).

6. Radisavljevic, B., Radenovic, A., Brivio, J., Giacometti, V. \& Kis, A. Nature Nanotech. 6, 147-150 (2011).

7. Radisavljevic, B., Whitwick, M. B. \& Kis, A. ACS Nano 5, 9934-9938 (2011).

8. Haigh, S. J. et al. Nature Mater. 11, 764-767 (2012).

9. Britnell, L. et al. Science 335, 947-950 (2012).

10. Novoselov, K. S. et al. Nature 490, 192-200 (2012).

11. Zeng, H., Dai, J., Yao, W., Xiao, D. \& Cui, X. Nature Nanotech. 7, 490-493 (2012).

12. Mak, K. F., He, K., Shan, J. \& Heinz, T. F. Nature Nanotech. 7, 494-496 (2012).

Corrected after print: 14 November 2012 


\section{Correction}

In the version of the Editorial 'Graphene is not alone' originally published

(Nature Nanotechnology 7, 683; 2012), the first name of the Review author was spelt incorrectly; it should have read 'Qing'.

Corrected in the HTML and PDF versions after print: 14 November 2012. 